\title{
SAMPLING MEASURES
}

\author{
JOAQUim OrTEGA-CERdì
}

\begin{abstract}
We give a description of all measures such that for any function in a weighted Fock spaces the $L^{p}$ norm with respect to the measure is equivalent to the usual norm in the space. We do so by a process of discretization that reduces the problem to the description of sampling sequences. The same kind of result holds for weighted Bergman spaces and the Paley-Wiener space.
\end{abstract}

\section{Introduction}

In this note, we address the following problem. Given a subharmonic function $\phi: \mathbb{C} \rightarrow \mathbb{R}$, under the restriction $m<\Delta \phi<M$, consider the weighted Fock space $\mathcal{F}_{\phi}^{p}(1 \leq p<\infty)$ consisting of entire functions $f$ such that

$$
\|f\|_{\phi, p}^{p}=\int_{\mathbb{C}}|f(z)|^{p} e^{-p \phi(z)} d m(z)<\infty
$$

equipped with its natural norm $\|\cdot\|_{\phi, p}$. The case of $p=2$ and $\phi=|z|^{2}$ corresponds to the classical Bargmann-Fock space. We want to describe the measures $\mu$ such that the norm of a function $f \in \mathcal{F}_{\phi}^{p}$ is equivalent to the original norm. That is, there is a constant $K>0$ such that,

$$
\frac{1}{K}\|f\|_{\phi, p}^{p} \leq \int_{\mathbb{C}}|f(z)|^{p} e^{-p \phi(z)} d \mu(z) \leq K\|f\|_{\phi, p}^{p} \quad \forall f \in \mathcal{F}_{\phi}^{p} .
$$

The inequality:

$$
\int_{\mathbb{C}}|f(z)|^{p} e^{-p \phi(z)} d \mu(z) \leq K\|f\|_{\phi, p}^{p} \quad \forall f \in \mathcal{F}_{\phi}^{p}
$$

Supported by the DGICYT grant PB95-0956-C02-02 and the CIRIT grant 1996SGR00026. 
is usually called a Carleson-type inequality (the original Carleson inequality was for $H^{p}$ functions in the disk) and the measures that satisfy such inequalities are called Carleson measures. We are interested in measures that satisfy both a direct and a reverse Carleson inequality. Such measures will be termed sampling measures. The description has already been achieved in the classical Bargmann-Fock space when $\mu$ consists of a sum of Dirac deltas in a remarkable work by Seip and Seip-Wallsten [Sei92], [SW92]. See also [BO95] and [OS98] for an extension to the case of weighted Fock spaces. Our work will be, in fact, a reduction of the general problem to the case of discrete measures.

Note that one can prove an analogous result for the weighted Bergman space since there is also a characterization of the sampling sequences in that setting (see [Sei93] for the usual Bergman space and [BO95] and [OS98] for the weighted case). Independently of this work, D. Luecking [Lue98] has reached also a different characterization of the sampling measures for the Bergman space. His characterization is in terms of properties of the support of the measures which are weak limits of translates of the original sampling measure. This is in the spirit of the work of Beurling [Beu89, p. 345] on the description of sampling sequences in the Bernstein space, which is also in the core of all of Seip's theorems mentioned above.

It is also possible to adapt the previous ideas to the Paley-Wiener space. We can describe all sampling measures in terms of the sampling sequences in a completely analogous way. In this setting there is an obvious extra difficulty. The sampling sequences are not characterized for $1<p<\infty$. Nevertheless, there are necessary and sufficient conditions which in some sense are very close. They are essentially due to Beurling, although he never explicitly stated them in the $L^{p}$ setting. The original Beurling theorem was stated for the Bernstein space $P W_{\tau}^{\infty}$ of entire functions of exponential type $\tau$ which are bounded on the real line. The details on how to get the $L^{p}$ variant can be found in [Sei95]. Also for a complete description of the sampling sequences in the Paley-Wiener space in the range $0<p \leq 1$ see [Flo98].

There is nothing special about dimension one. All our arguments can be reformulated in several variables. It is possible to describe all sampling measures in terms of sampling sequences. Unfortunately very little is known about sampling sequences in higher dimensions, thus this reduction is not very useful in that case.

Finally a word on notation. We write $f \lesssim g$ if there is a constant $K$ such that $f \leq K g$, and $f \simeq g$ if both $f \lesssim g$ and $g \lesssim f$. 


\section{Sampling measures in Fock space}

The description for an arbitrary measure will use the characterization of sampling sequences (i.e. sequences $\Gamma$ such that $\mu=\sum_{\gamma \in \Gamma} \delta_{\gamma}$ is a sampling measure) that was found in [OS98]:

Theorem (A). A sequence $\Gamma$ is a sampling sequence for the space $\mathcal{F}_{\phi}^{p}$ $(1 \leq p<\infty)$ if and only if the following two conditions are fulfilled

- $\Gamma$ is a finite union of uniformly separated sequences.

- There is a uniformly separated subsequence $\Gamma^{\prime} \subset \Gamma$ such that

$$
\liminf _{r \rightarrow \infty} \inf _{z \in \mathbb{C}} \frac{\#\left(D(z, r) \cap \Gamma^{\prime}\right)}{\int_{D(z, r)} \Delta \phi}>\frac{1}{2 \pi} .
$$

The first of the two conditions implies that $\mu$ is a Carleson measure for the Fock space and the second condition (the density condition) is needed in order to have a reverse Carleson inequality. In this description we could have used any compact $r K+z$ instead of the disk $D(z, r)$ under very mild assumptions on the boundary of $K$ (see [Lan67]). We could have taken a square for instance, which will be more convenient for the description in the case of general measures. In order to state the main result let us introduce some notation. We split the plane into a grid of squares of side length $r$. We take $N$ a large integer and $\delta>0$. We group together $N^{2}$ little squares of size $r$ forming a big square $S$ of side length $N r$. We denote by $n(S)$ the number of little squares $s$ of the original grid that are in $S$ such that $\mu(s) \geq \delta$. Of course, $n(S)$ does depend on $\delta, N, r$ and $S$. The theorem is the following:

Theorem. The measure $\mu$ is a sampling measure if and only if the following conditions are fulfilled

- There is a constant $C$ such that $\mu(D(z, 1)) \leq C$ for all disks of radius 1 .

- There is an $r>0$ and a grid made out of squares of side-length $r$, an integer $N>0$ and a positive $\delta>0$ such that

$$
\inf _{S} \frac{n(S)}{\int_{S} \Delta \phi}>\frac{1}{2 \pi}
$$

where the infimum is taken over all squares $S$ made out of $N^{2}$ little squares from the original grid. 
Before proving the theorem, let us note that if this density condition (1) holds for some $r, N, \delta$ it also holds for an arbitrary small $r$ (eventually decreasing $\delta$ and increasing $N$ ). We can also pick an arbitrary big $N$.

Note that condition (1) controls whether the measure $\mu$ is massive enough everywhere on a large scale. This control is done by taking into account averages of $\mu$ over very big squares $S$. Thus, if we have a sampling measure $\mu$ and we alter it by erasing its mass on an arbitrary big strip it will remain a sampling measure. On the other hand if we want $\mu$ to be a sampling measure it must be somehow spread out. In the case of the sum of Dirac deltas this follows because one considers separated sequences. In our case this is achieved by disregarding all little squares with small contribution of mass in the definition of $n(S)$.

As a corollary of this theorem we may give an alternative description of sampling measures in the particular situation when $d \mu(z)=\chi_{E}(z) d m(z)$, where $E$ is any measurable set in $\mathbb{C}$. This is an easier case that does not require the use of Beurling-type densities in order to be solved. It has, in fact, been considered previously by Logvinenko and Sereda in the Paley-Wiener space [VL74], by Luecking in the Bergman space [Lue81] and by Janson and Peetre in the Fock space [JPR87].

A measurable set $E \subset \mathbb{C}$ is said to be relatively dense in $\mathbb{C}$ if and only if there is a big $R>0$ such that $\inf _{z \in \mathbb{C}}|E \cap D(z, R)|>0$. The following corollary is a direct consequence of the theorem

Corollary (Janson-Peetre-Rochberg). A measurable set $E \subset \mathbb{C}$ satisfies

$$
\int_{\mathbb{C}}|f(z)|^{p} e^{-p \phi(z)} d m(z) \lesssim \int_{E}|f(z)|^{p} e^{-p \phi(z)} d m(z), \quad \forall f \in \mathcal{F}_{\phi}^{p},
$$

if and only if $E$ is relatively dense in $\mathbb{C}$.

Similarly we may obtain the Logvinenko-Sereda theorem for the PaleyWiener space [VL74] (in dimension 1) from Beurling description of sampling sequences in the Bernstein space $P W_{\tau}^{\infty}$.

Finally in the setting of the weighted Bergman space $B_{\alpha}^{p}$ of holomorphic functions in the unit disk, it is possible to reobtain Luecking's theorem [Lue81] from the description of the sampling sequences in $B_{\alpha}^{p}$ that was found in [Sei93].

In the proof of the theorem we will need the following lemma which plays the role of the Plancherel-Polya inequality in the case of PaleyWiener spaces and which is proved in [OS98]. 
Lemma. If $f$ belongs to $\mathcal{F}_{\phi}^{p}$, then

$$
\begin{aligned}
|f(z)|^{p} e^{-p \phi(z)} & \lesssim \int_{D(z, 1)}|f(w)|^{p} e^{-p \phi(w)} d m(w), \\
\left|\nabla\left(|f|^{p} e^{-p \phi}\right)(z)\right| & \lesssim \int_{D(z, 1)}|f(w)|^{p} e^{-p \phi(w)} d m(w)
\end{aligned}
$$

provided $f(z) \neq 0$.

The first condition $\mu(D) \leq C$ for all disks of radius 1 is equivalent to the Carleson inequality

$$
\int_{\mathbb{C}}|f(z)|^{p} e^{-p \phi(z)} d \mu(z) \lesssim\|f\|_{\phi, p}^{p} .
$$

Let us check that this condition is indeed sufficient. By the first inequality in the lemma, there is a constant $K$ such that

$$
\begin{aligned}
\int_{\mathbb{C}}|f(z)|^{p} e^{-p \phi(z)} d \mu(z) & \leq \int_{\mathbb{C}} K\left(\int_{D(z, 1)}|f(w)|^{p} e^{-p \phi(w)} d m(w)\right) d \mu(z) \\
& \leq C K \int_{\mathbb{C}}|f(w)|^{p} e^{-p \phi(w)} d m(w) .
\end{aligned}
$$

We have thus obtained a Carleson inequality. The smallest constant that appears in the Carleson inequality is called the Carleson constant and we have proved that it goes to 0 as $C=\sup _{z \in \mathbb{C}} \mu(D(z, 1))$ goes to 0 .

The condition is also necessary. If it did not hold, for any $\varepsilon>0$ and $n>0$, there would be disks $D\left(z_{n}, \varepsilon\right)$ with $\mu\left(D\left(z_{n}, \varepsilon\right)\right)>n$. This is not possible, since any single point $z_{n}$ is an interpolating sequence by itself (see [BO95]), therefore there are functions $f_{n}$ such that $\left\|f_{n}\right\|_{p, \phi} \lesssim 1$ and $\left|f_{n}\left(z_{n}\right)\right|^{p} e^{-p \phi\left(z_{n}\right)}=1$. Because of the inequality on the gradient in the lemma, the function $\left|f_{n}\right|^{p} e^{-p \phi}$ is close to 1 in an $\varepsilon$ neighborhood of $z_{n}$, thus $\int_{\mathbb{C}}\left|f_{n}\right|^{p} e^{-p \phi} d \mu \gtrsim n$ which is contradictory since we assumed that $\mu$ was a Carleson measure.

From now on, we will assume that the measure $\mu$ that we are considering satisfies $\mu(D(z, 1)) \leq C$. We will deal now with the density condition. 
Let us start by proving the necessity of (1). We pick a square grid of side length $r$ and for any $\delta>0$, we split the measure $\mu$ into two measures $\mu_{1}$ and $\mu_{2}$. The measure $\mu_{1}$ will be the original measure $\mu$ restricted to the squares $s$ such that $\mu(s) \geq \delta$ and $\mu_{2}$ will be the measure $\mu$ restricted to the squares $s$ such that $\mu(s)<\delta$. We start by proving that if $\delta$ is small enough then $\mu$ is a sampling measure if and only if $\mu_{1}$ is a sampling measure. The non-trivial direction is to check that $\mu_{1}$ is sampling when $\mu$ is sampling. This is the case because for any square $s$ on the grid we know that $\mu_{2}(s) /|s| \leq \delta / r^{2}$. Thus if we pick $\delta$ very small (with respect to $r$ ) we see that $\mu_{2}$ is a Carleson measure with a Carleson constant that goes to 0 as $\delta \rightarrow 0$. Therefore,

$$
\begin{aligned}
\int_{\mathbb{C}}|f|^{p} e^{-p \phi} d m & \lesssim \int_{\mathbb{C}}|f|^{p} e^{-p \phi} d \mu=\int_{\mathbb{C}}|f|^{p} e^{-p \phi} d \mu_{1}+\int_{\mathbb{C}}|f|^{p} e^{-p \phi} d \mu_{2} \\
& \leq \int_{\mathbb{C}}|f|^{p} e^{-p \phi} d \mu_{1}+\mathcal{O}(\delta) \int_{\mathbb{C}}|f|^{p} e^{-p \phi} d m .
\end{aligned}
$$

For a very small $\delta$ we can absorb the last summand in the left hand side of the inequality and we get

$$
\int_{\mathbb{C}}|f|^{p} e^{-p \phi} d m \lesssim \int_{\mathbb{C}}|f|^{p} e^{-p \phi} d \mu_{1}
$$

On the other hand, if the square grid has a very small length $r>0$, we may discretize the measure $\mu_{1}$ and still get a sampling measure. We set $\mu_{1}^{*}=\sum_{n} \mu_{1}\left(s_{n}\right) \delta_{a_{n}}$, where $s_{n}$ is any square of the grid and $a_{n}$ are the centers of the squares $s_{n}$. We are going to check that $\mu_{1}^{*}$ is sampling if and only if $\mu_{1}$ is sampling. We know that if $|z-w| \leq r<1 / 2$ then

$$
\left.|| f(z)\right|^{p} e^{-p \phi(z)}-\left.|f(w)|^{p} e^{-p \phi(w)}\left|\lesssim r \int_{D(z, 1)}\right| f(x)\right|^{p} e^{-p \phi(x)} d m(x)
$$

because of the inequality for the gradient in the lemma. Thus,

$$
\begin{aligned}
\int_{\mathbb{C}}|f|^{p} e^{-p \phi} d m & \lesssim \int_{\mathbb{C}}|f|^{p} e^{-p \phi} d \mu_{1} \\
& \lesssim \int_{\mathbb{C}}|f|^{p} e^{-p \phi} d \mu_{1}^{*}+\int_{\mathbb{C}} r \int_{D(z, 1)}|f(w)|^{p} e^{-p \phi(w)} d m(w) d \mu_{1}(z) \\
& \lesssim \int_{\mathbb{C}}|f|^{p} e^{-p \phi} d \mu_{1}^{*}+r \int_{\mathbb{C}}|f(w)|^{p} e^{-p \phi(w)} d m(w) .
\end{aligned}
$$


If $r$ is small enough, we absorb the last term on the left-hand side of the inequality. On the other direction the same is true, if $\mu_{1}^{*}$ is sampling we get

$$
\begin{aligned}
\int_{\mathbb{C}}|f|^{p} e^{-p \phi} d m & \lesssim \int_{\mathbb{C}}|f|^{p} e^{-p \phi} d \mu_{1}^{*} \\
& \lesssim \int_{\mathbb{C}}|f|^{p} e^{-p \phi} d \mu_{1}+\int_{\mathbb{C}} r \int_{D(z, 1)}|f(w)|^{p} e^{-p \phi(w)} d m(w) d \mu_{1}(z) \\
& \lesssim \int_{\mathbb{C}}|f|^{p} e^{-p \phi} d \mu_{1}+r \int_{\mathbb{C}}|f(w)|^{p} e^{-p \phi(w)} d m(w)
\end{aligned}
$$

and we conclude that $\mu_{1}$ is sampling as well.

Finally, since for any little square $s$ of the grid $\delta<\mu_{1}(s)<C$ or $\mu_{1}(s)=0$ we may conclude that the sequence $\Gamma$ made out of the centers of the squares where $\mu_{1}$ has mass is a sampling sequence if and only if $\mu_{1}^{*}$ is a sampling measure and we get the theorem thanks to the characterization of the sampling sequences (Theorem A).

Acknowledgement. I am grateful to the referee for some appropriate remarks that have improved the readability of this note.

\section{References}

[Beu89] A. Beurling, "The collected works of Arne Beurling," vol. 2, Birkhäuser, Boston, 1989.

[BO95] B. Berndtsson And J. OrtegA-Cerdà, Interpolation and sampling in Hilbert spaces of holomorphic functions, J. Reine Angew. Math. 464 (1995), 109-120.

[Flo98] K. M. Flornes, Sampling and interpolation in the PaleyWiener spaces $L_{\pi}^{p}, 0<p \leq 1$, Publ. Mat. 42 (1998), 103-118.

[JPR87] S. Janson, J. Peetre And R. RochberG, Hankel forms and the Fock space, Rev. Mat. Iberoamericana 3(1) (1987), 61-138.

[Lan67] H. J. LANDAU, Necessary density conditions for sampling and interpolation of certain entire functions, Acta Math. 117 (1967), $37-52$.

[Lue81] D. H. LueCKING, Inequalities on Bergman spaces, Illinois J. Math. 25(1) (1981), 1-11.

[Lue98] D. H. Luecking, Sampling measures for the Bergman space on the unit disk, Preprint (1998). 
[OS98] J. Ortega-Cerdà and K. Seip, Beurling-type density theorems for weighted $L^{p}$ spaces of entire functions, J. Anal. Math. (1998) (to appear).

[Sei92] K. SEIP, Density theorems for sampling and interpolation in the Bargmann-Fock space I, J. Reine Angew. Math. 429 (1992), 91-106.

[Sei93] K. SEIP, Beurling type density theorems in the unit disk, Invent. Math. 113 (1993), 21-39.

[Sei95] K. SEIP, On the connection between exponential bases and certain related sequences in $L^{2}(-\pi, \pi), J$. Funct. Anal. 130 (1995), 131-160.

[SW92] K. Seip and R. Wallstén, Density theorems for sampling and interpolation in the Bargmann-Fock space II, J. Reine Angew. Math. 429 (1992), 107-113.

[VL74] Y. F. SEREDA AND V. N. LogVinenKo, Equivalence of norms in spaces of entire functions of exponential type, Teor. Funktsi乞 Funktsional Anal. i Prilozhen 20 (1974), 62-78.

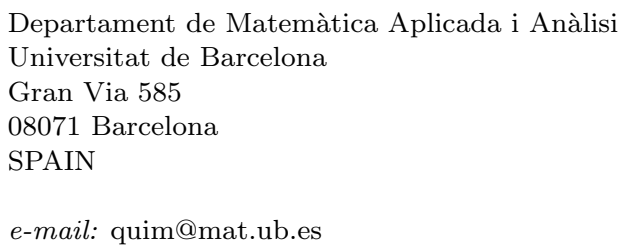

Primera versió rebuda el 19 de maig de 1998, darrera versió rebuda el 9 d'octubre de 1998 